\title{
HAPLOTYPES AND ALLELIC FREQUENCIES OF 12 Y-STR LOCI IN MONGOLIAN AND KOREAN MALE GROUPS
}

\author{
B. Dashnyam ${ }^{1}$, D.Bayarlkhagva ${ }^{2}$, Evgeniy Namdakov ${ }^{1}$, G.Batjil ${ }^{3}, S$. Ganbold $^{4}$ \\ ${ }^{1}$ Монгол олон улсын дээд сургууль, \\ ${ }^{2}$ Биологийн факультет, МУИС, \\ ${ }^{3}$ Батлан Хамгаалахын Эмгэг Судлал, ШҮYх Эмнэлэг, \\ ${ }^{4}$ Биологийн лаборатори, ШҮҮхийн Шинжилгээний Үндэсний Хүрээлэн
}

\begin{abstract}
Уг судалгааг “Монгол хүний Ү хромосомын генетик мэдээллийн сан”, “Цэргийн албан хаагчдын генийн мэдээллийн сан бүрдүҮлэх, туриилт судалгаа“" төслүүдийн хүрээнд хийж гүйцээтаэв.
\end{abstract}

\section{INTRODUCTION}

DNA nucleotide sequences would never be completely repeated in human populations except the case of identical twins, and so everyone has their own DNA sequence. That's why DNA analysis is useful for human identification. Nowadays there are several methods for DNA diversity analysis such as Restriction Fragment Length Polymorphism (RFLP), Single Nucleotide Polymorphism (SNP) analysis, Polymerase Chain Reaction (PCR), Mitochondrial DNA Analysis, and Y-Chromosome Analysis.

STR analysis is widely used by the FBI and Interpol for distinguishing one DNA profile from another. In the USA, the FBI uses 13 specific well-known STR regions of DNA for a Combined DNA Index System (CODIS). The probability that two individuals will have the same 13-loci DNA profile are about one in a billion cases (http://www.fbi.gov/hq/lab/ $\mathrm{html} /$ codisbrochure text.htm). In the USA the core loci are CSF1PO, FGA, THO1, TPOX, vWA, D3S1358, D5S818, D7S820, D8S1179, D13S317, D16S539, D21S11, and Amelogenin as an optional locus for determining the gender. In the UK the basic loci are FGA, THO1, vWA, D2S1338, D3S1358, D8S1179, D16S529, D18S51, D19S433, D21S11, and Amelogenin as an optional locus (http://www.cstl.nist.gov/ strbase/coreSTRs.htm).

In this work we used $\mathrm{Y}$ chromosome Short Tandem Repeats (STRs) analysis. STRs or microsatellites are repeats of nucleotides in DNA. They contain from 2 to 6 nucleotides repeated. And because of these, STRs can be easily amplified by PCR and then analyzed.

Y-STR analysis is important not only in the study of evolution (Hammer et al., 1997, Su et al., 1999, Kayser et al., 2001) or paternal lineages (Hammer, 1995, Jobling and TylerSmith, 1995, Underhill et al., 2001), but also in forensic cases ( Gill et al., 2001), because sometimes forensic expert could have mixed DNA samples from a man and woman. Also, the $\mathrm{Y}$ chromosome has a haploid state and is transmitted from father to son (Kwak et al., 2005). In this case, Y-STR analysis is a very common method for determining a man's DNA profile.

The results of Y-STR analysis lead to the construction of Y-STR haplotypes specific for individuals. A haplotype is a combination of alleles on the same chromosome, in our case on the Y-chromosome. Derenko et al. (2004) mentioned that Mongols are at the middle position between the nations of South Siberia and Central/East Asia. Data analysis of the pairwise $\varphi_{\text {st }}$ distances (Derenko et al., 2004) and of seven Y chromosome binary markers (Jin and Kim, 2003) has shown that Koreans are more closely related to Northern Chinese than to Mongols. Kwak et al. (2005) tested ten ethnic groups from East Asia and pointed out that all of the examined people have high haplotype diversity $(\geq 0.997)$ by analysis of 11 Y-STR loci.

Perhaps, Mongolian and Korean male groups could have high allelic numbers of 12 Y-STR loci: DYS391, DYS389I, DYS439, 
DYS389II, DYS438, DYS437, DYS19, DYS392, DYS393, DYS390, DYS385 a/b. And that's why we tried to look at those frequencies for highlighting possible interactions between those two different nationalities.

\section{MATERIAL AND METHODS}

In this experiment we used cheek swab or saliva collected by cotton sticks from 15 Mongols and 15 Koreans which were not related to each other and worked or studied at MIU. DNA extraction and Agarose Gel Electrophoresis were carried out at "ГИСТОГЕН" (GISTOGEN) laboratory in the Bayanzurkh district, and PCR amplification and analyses of PCR products were conducted in the Biology laboratory of the National Institute of Forensic Medicine, Ulaanbaatar, Mongolia

After collecting saliva and cheek swabs were stored at $-20^{\circ} \mathrm{C}$ for two months. Then we extracted DNA by using the phenolchlorophorm-isoamyl alcohol standard method (Sambrook et al., 1989). Firstly, we added lysis buffer $(500 \mu \mathrm{L})$ and proteinase $\mathrm{K}(40 \mu \mathrm{L})$ and then incubated it for one hour at $+56^{0}$ with periodic shaking. Next, we collected all liquid from the tubes and transferred it to new tubes. Then, we added $600 \mu \mathrm{L}$ of phenol-chlorophormisoamyl alcohol $(25: 24: 1, \mathrm{~V} / \mathrm{V})$ to the extract and centrifuged for 5 minutes at 12000 RPM. The supernatant (upper layer) was collected and chlorophorm-isoacrylamyl $600 \mu \mathrm{L}$ (24:1) added for purification by separating DNA from other macromolecules, and centrifuged for 5 minutes at 12000 RPM. Then the supernatant was collected and $800 \mu \mathrm{L}$ of $96 \%$ ethyl alcohol added and kept at $-20^{\circ} \mathrm{C}$ for one hour. Next we centrifuged the solution for 5 minutes at 12000 RPM, poured out the ethyl alcohol (96\%) from the tubes, leaving DNA precipitate in the tube and added $70 \%$ ethanol and then again centrifuged for 5 minutes at 12000 RPM. Finally, we poured out the ethanol and dried the tubes for 30 minutes at $56^{\circ} \mathrm{C}$.

Afterward, we added $50 \mu \mathrm{L}$ NucleaseFree Water (Promega Corp.) and incubated at $+55^{\circ} \mathrm{C}$ in a water bath for $20-30$ minutes. And then, started $8 \%$ agarose gel electrophoresis by mixing DNA samples and loading solution. Electrophoresis was needed to determine the amount of DNA.

Multiplex PCR amplification and detection of the amplified product were conducted by following the instructions described by Kwak et al (2005).

Multiplex PCR amplifications were performed by mixing approximately $5 \mu \mathrm{g}$ of genomic DNA, 1.5 U AmpliTaq Gold DNA polymerase, $200 \mu \mathrm{mol} / \mathrm{L} \mathrm{dNTPs}, 2.0 \mathrm{mmol} / \mathrm{L}$ of $\mathrm{MgCl}_{2}$, TRIS- $\mathrm{HCl}(\mathrm{pH} 8.3) 10 \mathrm{mmol} / \mathrm{L}$, and $50 \mathrm{mmol} / \mathrm{L}$ of $\mathrm{KCl}$ (all from Applied BioSystems Corp.). Amplification reactions were carried out in Thermal Cycler 9700 (Applied BioSystems), with standard settings: multiplex GK1: initial denaturation at $95^{\circ} \mathrm{C}$ for $10 \mathrm{~min}$, followed by 35 cycles of $94^{\circ} \mathrm{C}$ for 1 $\min , 54^{\circ} \mathrm{C}$ for $2 \mathrm{~min}, 72^{\circ} \mathrm{C}$ for $2 \mathrm{~min}$, and a final extension at $60^{\circ} \mathrm{C}$ for $40 \mathrm{~min}$; multiplex GK2: initial denaturation at $95^{\circ} \mathrm{C}$ for $10 \mathrm{~min}$, followed by 35 cycles of $94^{\circ} \mathrm{C}$ for $1 \mathrm{~min}, 57^{\circ} \mathrm{C}$ for $1 \mathrm{~min}, 72^{\circ} \mathrm{C}$ for $1.5 \mathrm{~min}$, and final extension at $60^{\circ} \mathrm{C}$ for $30 \mathrm{~min}$.

Detection of amplified product was accomplished in an ABI 310 Prism Genetic Analyzer (Applied BioSystems) by mixing with Hi-Di Formamide and LYS size standard with PCR product and putting to the capillary array for $5 \mathrm{~s}$ at $15,000 \mathrm{~V}$. Separations were performed at $15,000 \mathrm{~V}$ for 34 min using the POP-4 polymer (Applied BioSystems, P/N 402838), 14 Genetic Analyzer Buffer with EDTA (P/N 402824), and a 47-cm array (P/N 402839) with a run temperature of $60^{\circ} \mathrm{C}$. Following data collection, samples were analyzed with GeneMapper 3.1 Software (Applied BioSystems) following the manufacturer's instructions.

\section{RESULTS AND DISCUSSION}

All of the studied males of Korean and Mongolian ethnic groups had different haplotypes (see tables 1 and 2). In comparison with Genghis Khan's star cluster (Zerjal et al., 
2003) which has 9 loci presented in this work except DYS19 and DYS 385a/b, one person has the similar haplotype (\#15 in the table 2) and one person has almost the same haplotype just with one exception on DYS437 (\#8 in the table 2). The rest of the people studied had three or more differences. According to loci presented in our research, Genghis Khan's haplotype is 10-13-10-29-10-14-11-13-25 corresponding to loci DYS391-DYS389I-DYS439-DYS438DYS437-DYS392-DYS393-DYS390.

Table 1

Haplotypes of Korean male group where $N$ is the number of haplotypes

\begin{tabular}{|c|c|c|c|c|c|c|c|c|c|c|c|c|}
\hline No & $\mathbf{N}$ & $\begin{array}{l}\overline{\tilde{m}} \\
\hat{2} \\
\text { हो }\end{array}$ & 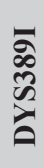 & 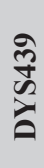 & $\begin{array}{l}\bar{\sigma} \\
\infty \\
\tilde{D} \\
\hat{\partial} \\
0\end{array}$ & 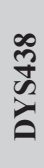 & \begin{tabular}{l}
$\hat{0}$ \\
\multirow{2}{*}{} \\
0
\end{tabular} & $\begin{array}{l}\frac{\partial}{2} \\
\frac{\lambda}{2}\end{array}$ & $\begin{array}{l}\text { है } \\
\text { हે } \\
\text { है }\end{array}$ & 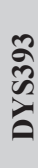 & 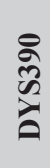 & 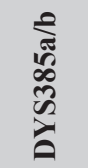 \\
\hline 1 & 1 & 10 & 14 & 12 & 30 & 13 & 14 & 16 & 14 & 13 & 23 & $10-18$ \\
\hline 2 & 1 & 10 & 12 & 13 & 28 & 10 & 15 & 17 & 13 & 13 & 24 & $14-20$ \\
\hline 3 & 1 & 10 & 12 & 12 & 30 & 10 & 14 & 17 & 14 & 12 & 24 & $14-17$ \\
\hline 4 & 1 & 10 & 14 & 11 & 29 & 13 & 14 & 16 & 13 & 13 & 23 & $10-19$ \\
\hline 5 & 1 & 10 & 14 & 12 & 29 & 10 & 14 & 16 & 11 & 14 & 21 & $11-16$ \\
\hline 6 & 1 & 10 & 12 & 13 & 28 & 10 & 14 & 15 & 10 & 12 & 25 & $12-18$ \\
\hline 7 & 1 & 10 & 13 & 12 & 29 & 13 & 14 & 16 & 13 & 13 & 24 & $10-18$ \\
\hline 8 & 1 & 10 & 13 & 12 & 29 & 9 & 14 & 16 & 11 & 14 & 23 & 14-17 \\
\hline 9 & 1 & 10 & 12 & 12 & 27 & 13 & 14 & 15 & 13 & 13 & 24 & $10-18$ \\
\hline 10 & 1 & 10 & 12 & 12 & 30 & 10 & 14 & 17 & 12 & 12 & 24 & $12-17$ \\
\hline 11 & 1 & 10 & 14 & 10 & 30 & 10 & 14 & 15 & 11 & 15 & 23 & $11-20$ \\
\hline 12 & 1 & 11 & 13 & 10 & 29 & 11 & 14 & 16 & 11 & 14 & 24 & $11-24$ \\
\hline 13 & 1 & 10 & 12 & 11 & 27 & 11 & 15 & 14 & 14 & 12 & 24 & $13-19$ \\
\hline 14 & 1 & 10 & 12 & 11 & 29 & 10 & 14 & 17 & 13 & 12 & 24 & $14-21$ \\
\hline 15 & 1 & 10 & 12 & 12 & 27 & 11 & 15 & 15 & 14 & 12 & 25 & $12-19$ \\
\hline
\end{tabular}

Table 2

Haplotypes of Mongolian male group where $N$ is the number of haplotypes

\begin{tabular}{|c|c|c|c|c|c|c|c|c|c|c|c|c|}
\hline No & $\mathbf{N}$ & 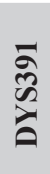 & 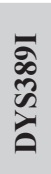 & $\begin{array}{l}\text { के } \\
\text { है } \\
\text { है }\end{array}$ & 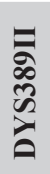 & 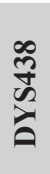 & $\begin{array}{l}\hat{\infty} \\
\text { है } \\
\text { है }\end{array}$ & $\begin{array}{l}\frac{\partial}{\sqrt{2}} \\
\frac{1}{0}\end{array}$ & 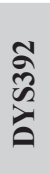 & 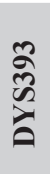 & 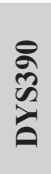 & $\begin{array}{l}\sum_{\tilde{W}} \\
\infty \\
\infty \\
\tilde{\nu} \\
0\end{array}$ \\
\hline 1 & 1 & 9 & 13 & 11 & 29 & 10 & 14 & 14 & 10 & 13 & 24 & $12-12$ \\
\hline 2 & 1 & 11 & 13 & 11 & 29 & 11 & 14 & 14 & 12 & 13 & 23 & $11-12$ \\
\hline 3 & 1 & 10 & 12 & 12 & 27 & 10 & 15 & 15 & 12 & 12 & 23 & $12-18$ \\
\hline 4 & 1 & 10 & 13 & 10 & 29 & 10 & 14 & 14 & 11 & 12 & 23 & 14-18 \\
\hline 5 & 1 & 10 & 14 & 11 & 29 & 11 & 16 & 15 & 10 & 14 & 23 & $13-19$ \\
\hline 6 & 1 & 11 & 13 & 10 & 29 & 11 & 14 & 16 & 11 & 14 & 24 & $11-14$ \\
\hline 7 & 1 & 10 & 12 & 13 & 29 & 11 & 17 & 15 & 11 & 12 & 25 & $10-18$ \\
\hline 8 & 1 & 10 & 13 & 10 & 29 & 10 & 15 & 16 & 11 & 13 & 25 & $12-13$ \\
\hline 9 & 1 & 10 & 13 & 13 & 29 & 10 & 17 & 14 & 11 & 12 & 23 & $9-18$ \\
\hline 10 & 1 & 10 & 13 & 12 & 29 & 11 & 15 & 14 & 14 & 12 & 23 & $13-18$ \\
\hline 11 & 1 & 10 & 12 & 12 & 29 & 10 & 14 & 17 & 13 & 12 & 26 & $11-21$ \\
\hline 12 & 1 & 12 & 14 & 11 & 31 & 11 & 14 & 17 & 11 & 13 & 24 & $12-12$ \\
\hline 13 & 1 & 9 & 13 & 12 & 30 & 10 & 14 & 14 & 11 & 13 & 23 & $10-11$ \\
\hline 14 & 1 & 11 & 12 & 11 & 28 & 11 & 16 & 14 & 14 & 8 & 24 & $13-13$ \\
\hline 15 & 1 & 10 & 13 & 10 & 29 & 10 & 14 & 16 & 11 & 13 & 25 & $12-13$ \\
\hline
\end{tabular}


We compared Korean and Mongolian male groups with French (Roewer et al., 2005), Egyptian (Manni et al., 2002) and Aasiaatian (Hallenberg et al., 2009) populations. French people represent a European nation, Egyptians - Northern African nation, and Aasiaats are one of the native North American peoples living in Greenland. The haplotypes of French, Egyptian, and Aasiaat male groups are shown in Tables 3, 4, and 5, respectively. Those results show that all of five ethnic groups, i.e. Koreans, Mongols, French, Egyptians, and Aasiaats, have different haplotypes.

Table 3

Haplotypes of French male groups where $N$ is the number of haplotypes and (-) is unknown data

\begin{tabular}{|c|c|c|c|c|c|c|c|c|c|c|c|}
\hline $\mathbf{N}$ & $\begin{array}{l}\overline{\hat{\sigma}} \\
\hat{0} \\
\overline{0}\end{array}$ & 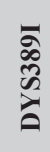 & $\begin{array}{l}\hat{\tilde{y}} \\
\text { क्रे } \\
0\end{array}$ & 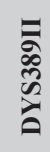 & 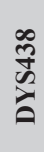 & 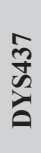 & $\begin{array}{l}\overrightarrow{\bar{D}} \\
\vec{\partial}\end{array}$ & 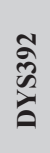 & $\begin{array}{l}0 \\
\hat{n} \\
0 \\
0\end{array}$ & 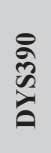 & 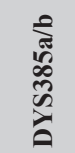 \\
\hline 3 & 11 & 14 & - & 30 & - & - & 14 & 13 & 13 & 24 & $11-14$ \\
\hline 2 & 11 & 13 & - & 29 & - & - & 14 & 13 & 13 & 24 & $12-14$ \\
\hline 2 & 11 & 13 & - & 30 & - & - & 14 & 13 & 13 & 24 & $11-14$ \\
\hline 2 & 10 & 13 & - & 31 & - & - & 14 & 11 & 12 & 23 & $13-15$ \\
\hline 2 & 10 & 13 & - & 31 & - & - & 14 & 11 & 12 & 23 & $13-16$ \\
\hline 1 & 11 & 13 & - & 29 & - & - & 12 & 13 & 14 & 22 & $13-15$ \\
\hline 1 & 10 & 13 & - & 30 & - & - & 12 & 11 & 15 & 21 & $16-17$ \\
\hline 1 & 10 & 14 & - & 30 & - & - & 12 & 13 & 13 & 24 & $13-14$ \\
\hline 1 & 12 & 14 & - & 30 & - & - & 12 & 14 & 13 & 24 & $12-14$ \\
\hline 1 & 10 & 14 & - & 32 & - & - & 12 & 11 & 13 & 25 & $16-17$ \\
\hline
\end{tabular}

Table 4

Haplotypes of Egyptian male groups where $N$ is the number of haplotypes and (-) is unknown data

\begin{tabular}{|c|c|c|c|c|c|c|c|c|c|c|c|}
\hline $\mathbf{N}$ & $\begin{array}{l}\overline{\tilde{\partial}} \\
\hat{\sigma} \\
0\end{array}$ & 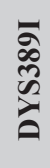 & 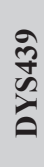 & 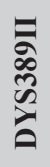 & 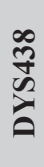 & $\begin{array}{l}\hat{\tilde{f}} \\
\text { के } \\
\text { है }\end{array}$ & $\begin{array}{l}\overrightarrow{\bar{\pi}} \\
\vec{\lambda}\end{array}$ & 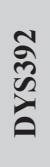 & 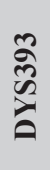 & 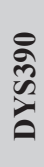 & 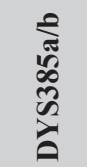 \\
\hline 2 & 10 & 12 & - & 29 & - & - & 13 & 11 & 13 & 23 & $15-18$ \\
\hline 2 & 9 & 13 & - & 29 & - & - & 13 & 11 & 13 & 23 & $13-15$ \\
\hline 2 & 10 & 13 & - & 29 & - & - & 14 & 11 & 12 & 25 & $11-18$ \\
\hline 2 & 10 & 12 & - & 29 & - & - & 16 & 12 & 12 & 23 & $14-17$ \\
\hline 1 & 9 & 13 & - & 28 & - & - & 13 & 11 & 13 & 23 & $14-16$ \\
\hline 1 & 9 & 13 & - & 29 & - & - & 13 & 11 & 13 & 21 & $15-17$ \\
\hline 1 & 9 & 12 & - & 28 & - & - & 13 & 11 & 11 & 22 & $17-17$ \\
\hline 1 & 10 & 13 & - & 29 & - & - & 13 & 14 & 13 & 23 & $14-16$ \\
\hline 1 & 9 & 13 & - & 29 & - & - & 13 & 11 & 13 & 24 & $13-14$ \\
\hline 1 & 10 & 13 & - & 29 & - & - & 13 & 11 & 13 & 24 & $13-15$ \\
\hline
\end{tabular}


Haplotypes of Aasiaat male groups where $N$ is the number of haplotypes

\begin{tabular}{|c|c|c|c|c|c|c|c|c|c|c|c|}
\hline $\mathbf{N}$ & $\begin{array}{l}\bar{\sigma} \\
\text { हे } \\
\text { है }\end{array}$ & $\begin{array}{l}\bar{\sigma} \\
\infty \\
\tilde{\infty} \\
\hat{0} \\
0\end{array}$ & $\begin{array}{l}\text { हे } \\
\text { ஸे } \\
\text { है }\end{array}$ & $\begin{array}{l}\bar{\sigma} \\
\infty \\
\tilde{N} \\
\tilde{\lambda} \\
0\end{array}$ & 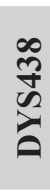 & 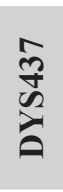 & 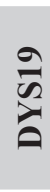 & 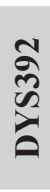 & $\begin{array}{l}\text { బิ } \\
\text { है } \\
\text { है }\end{array}$ & 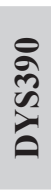 & 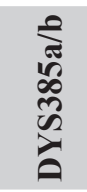 \\
\hline 2 & 10 & 14 & 11 & 30 & 11 & 15 & 13 & 15 & 14 & 24 & $13-20$ \\
\hline 2 & 10 & 14 & 11 & 30 & 11 & 15 & 13 & 15 & 14 & 24 & $13-21$ \\
\hline 2 & 10 & 14 & 13 & 31 & 11 & 15 & 13 & 14 & 13 & 24 & $14-18$ \\
\hline 2 & 10 & 14 & 13 & 31 & 10 & 15 & 13 & 14 & 14 & 24 & $16-18$ \\
\hline 2 & 10 & 15 & 13 & 32 & 11 & 15 & 13 & 14 & 13 & 24 & $14-17$ \\
\hline 2 & 10 & 12 & 11 & 28 & 10 & 16 & 15 & 11 & 13 & 23 & 14-14 \\
\hline 1 & 10 & 14 & 11 & 30 & 11 & 15 & 13 & 15 & 14 & 24 & $13-22$ \\
\hline 1 & 11 & 14 & 11 & 30 & 11 & 14 & 13 & 15 & 14 & 24 & $13-20$ \\
\hline 1 & 10 & 14 & 11 & 30 & 11 & 15 & 13 & 15 & 14 & 25 & $13-18$ \\
\hline 1 & 10 & 14 & 12 & 31 & 11 & 15 & 13 & 14 & 13 & 24 & $14-17$ \\
\hline
\end{tabular}

Allelic frequencies among Mongols on two loci: DYS438 and DYS392 (Table 7). and Koreans show that those two groups At four loci: DYS393, DYS390, DYS385a and have many differences. As shown in Table 6, DYS385b, Koreans and Mongols have different Mongols have more alleles of STRs on three alleles and different allelic frequencies as loci: DYS391, DYS389II, and DYS437. shown in Table 8.

However, Koreans have more alleles of STRs

Table 6

Mongols have more alleles than Koreans at two loci

\begin{tabular}{|c|c|c|c|c|c|c|}
\hline & \multicolumn{2}{|c|}{ DYS391 } & \multicolumn{2}{|c|}{ DYS389II } & \multicolumn{2}{|c|}{ DYS437 } \\
\hline & Allele & Frequency & Allele & Frequency & Allele & Frequency \\
\hline \multicolumn{7}{|c|}{ Koreans } \\
\hline & 10 & $93.3 \%$ & 27 & $20 \%$ & 14 & $66.7 \%$ \\
\hline & 11 & $6.7 \%$ & 28 & $13.3 \%$ & 15 & $26.7 \%$ \\
\hline & & & 29 & $40 \%$ & 16 & $6.7 \%$ \\
\hline & & & 30 & $26.7 \%$ & & \\
\hline \multicolumn{7}{|c|}{ Mongols } \\
\hline & 9 & $13.3 \%$ & 27 & $6.7 \%$ & 14 & $53.3 \%$ \\
\hline & 10 & $60 \%$ & 28 & $6.7 \%$ & 15 & $20 \%$ \\
\hline & 11 & $20 \%$ & 29 & $66.7 \%$ & 16 & $13.3 \%$ \\
\hline & 12 & $6.7 \%$ & 30 & $13.3 \%$ & 17 & $13.3 \%$ \\
\hline & & & 31 & $6.7 \%$ & & \\
\hline
\end{tabular}

Table 7

Koreans have more alleles of STRs than Mongols

\begin{tabular}{|c|c|c|c|}
\hline \multicolumn{2}{|c|}{ DYS438 } & \multicolumn{2}{c|}{ DYS392 } \\
Allele & $\begin{array}{c}\text { Frequency } \\
\text { Koreans }\end{array}$ & Allele & Frequency \\
\hline 9 & $6.7 \%$ & 10 & $6.7 \%$ \\
\hline 10 & $46.7 \%$ & 11 & $20 \%$ \\
\hline 11 & $20 \%$ & 12 & $6.7 \%$ \\
\hline 13 & $26.7 \%$ & 13 & $33.3 \%$ \\
\hline & & 14 & $26.7 \%$ \\
\hline & & 15 & $6.7 \%$ \\
\hline
\end{tabular}

\begin{tabular}{|c|c|c|c|}
\hline \multicolumn{2}{|c|}{ DYS438 } & \multicolumn{2}{c|}{ DYS392 } \\
\hline Allele & $\begin{array}{c}\text { Frequency } \\
\text { Mongols }\end{array}$ & Allele & Frequency \\
\hline 10 & $53.3 \%$ & 10 & $13.3 \%$ \\
\hline 11 & $46.7 \%$ & 11 & $53.3 \%$ \\
\hline & & 12 & $13.3 \%$ \\
& & 13 & $6.7 \%$ \\
\hline & & 14 & $13.3 \%$ \\
\hline
\end{tabular}


Table 8

Koreans and Mongols have different alleles and different allele frequencies

\begin{tabular}{|c|c|c|c|c|c|c|}
\hline & \multicolumn{2}{|c|}{ DYS393 } & \multicolumn{2}{|c|}{ DYS390 } & \multicolumn{2}{|c|}{ DYS385 a/b } \\
\hline & Allele & Frequency & Allele & Frequency & Allele & Frequency \\
\hline \multicolumn{7}{|c|}{ Koreans } \\
\hline & 12 & $46.7 \%$ & 21 & $6.7 \%$ & 10 & $13.3 \%$ \\
\hline & 13 & $33.3 \%$ & 23 & $26.7 \%$ & 11 & $6.7 \%$ \\
\hline & 14 & $13.3 \%$ & 24 & $53.3 \%$ & 12 & $10 \%$ \\
\hline 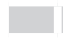 & 15 & $6.7 \%$ & 25 & $13.3 \%$ & 13 & $3.3 \%$ \\
\hline & & & & & 14 & $16.7 \%$ \\
\hline 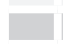 & & & & & 16 & $3.3 \%$ \\
\hline & & & & & 17 & $10 \%$ \\
\hline 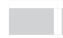 & & & & & 18 & $13.3 \%$ \\
\hline & & & & & 19 & $13.3 \%$ \\
\hline 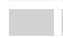 & & & & & 20 & $6.7 \%$ \\
\hline & & & & & 21 & $3.3 \%$ \\
\hline \multicolumn{7}{|c|}{ Mongols } \\
\hline & 8 & $6.7 \%$ & 23 & $46.7 \%$ & 9 & $3.3 \%$ \\
\hline 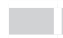 & 12 & $40 \%$ & 24 & $26.7 \%$ & 10 & $6.7 \%$ \\
\hline & 13 & $40 \%$ & 25 & $20 \%$ & 11 & $13.3 \%$ \\
\hline 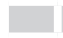 & 14 & $13.3 \%$ & 26 & $6.7 \%$ & 12 & $26.7 \%$ \\
\hline & & & & & 13 & $20 \%$ \\
\hline 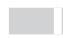 & & & & & 14 & $6.7 \%$ \\
\hline & & & & & 18 & $16.7 \%$ \\
\hline 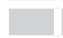 & & & & & 19 & $3.3 \%$ \\
\hline & & & & & 21 & $3.3 \%$ \\
\hline
\end{tabular}

However, Mongolian and Korean Moreover, Mongols and Koreans could have populations have the same alleles of STRs at close genetic relationships by having one main three loci: DYS389I, DYS439, and DYS19; allele at four loci with higher frequency as but with different allelic frequencies (Table 9). shown in Table 10.

Table 9

Mongols have the same alleles of STRs as Koreans at three loci but allele frequencies differ

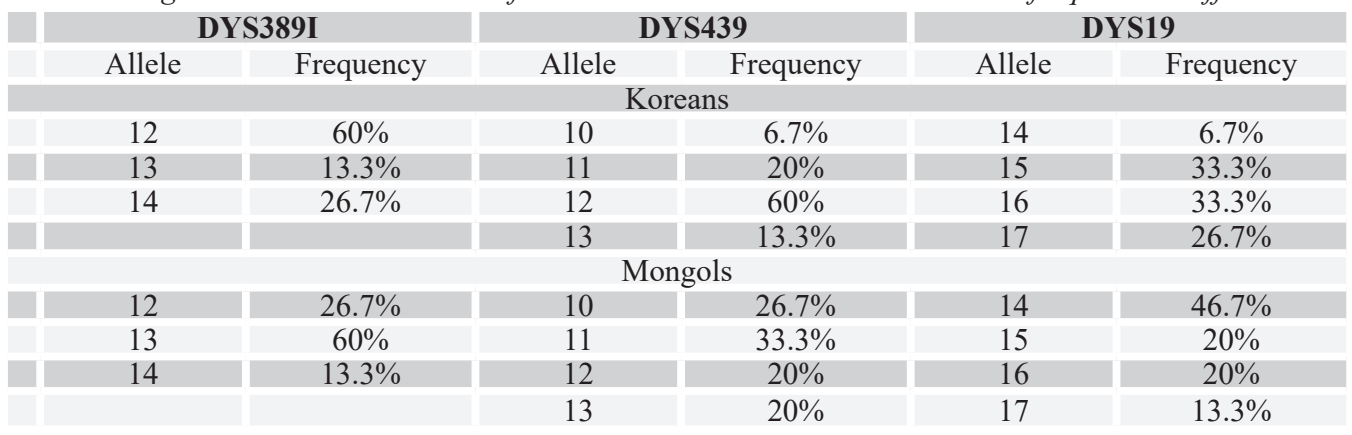

Table 10

Koreans and Mongols have one main allele at four loci with higher frequency

\begin{tabular}{|c|c|c|c|c|}
\hline & \multicolumn{3}{|c|}{ Koreans } & \multicolumn{3}{c|}{ Mongols } \\
\hline DYS391 & Allele & Frequency & Allele & Frequency \\
\hline DYS389II & 10 & $93.3 \%$ & 10 & $60 \%$ \\
\hline DYS437 & 29 & $40 \%$ & 29 & $66.7 \%$ \\
\hline DYS438 & 14 & $66.7 \%$ & 14 & $53.3 \%$ \\
\hline
\end{tabular}




\section{CONCLUSION}

Koreans and Mongolian populations show unique haplotypes which can mean the early population growth. By comparing Mongolian and Korean haplotypes with Genghis Khan's star cluster, we could say that some Mongols have a tendency to be close to Genghis Khan's cluster, but Koreans do not have that tendency. And in a comparison of three populations (French, Egyptian, and Aasiaat) from different continents (Europe, Africa, and North America respectively) with Korean and Mongolian ethnic groups, we could say that all of them differ from each other.

Koreans and Mongols have more differences than similarities by allelic frequencies. However, we could observe the tendency that Korean and Mongolian ethnic groups might have some genetic relationships in the past by having one common ancestor, but by the time their haplotypes mutated, because they have some similarities as described in Tables 9 and 10 .

\section{REFERENCES}

1. CODIS Combined DNA Index System, http://www.fbi.gov/hq/lab/html/codisbrochure_text. htm, updated 2009.

2. Core STR Loci Used in Human Identity Testing, http://www.cstl.nist.gov/strbase/coreSTRs. htm, 2006.

3. Derenko MV, Lunkina AV, Malyarchuk BA, Zakharov IA, Tsedev Ts, Park KS, Cho YM, Lee HK, Chu Ch.H. 2004. Restriction Polymorphism of Mitochondrial DNA in Koreans and Mongolians. Russian Journal of Genetics 40/11: 1292-1299.

4. FBI CODIS Core STR Loci, http://www.cstl.nist.gov/strbase/fbicore.htm, updated 2006.

5. Gill P, Brenner C, Brinkmann B et al. 2001. DNA Commission of the International Society of Forensic Genetics: recommendations on forensic analysis using Y-chromosome STRs. Int J Leg Med 114:305-309.

6. Hallenberg C., Tomas C., Simonsen B., Morling N. 2009. 'Y-chromosome STR haplotypes in males from Greenland', Forensic Science International: Genetics 3(4), e145-e146.

7. Hammer MF. 1995. A recent common ancestry for human Y chromosomes. Nature 378:376378.

8. Hammer MF, Spurdle AB, Karafet T, Bonner MR, Wood ET, Novelletto A, Malaspina P, Mitchell RJ, Horai S, Jenkins T, Zegura SL. 1997. The geographic distribution of human Y chromosome variation. Genetics 145:787-805.

9. Jin HJ and Kim W. 2003. Genetic Relationship between Korean and Mongolian Populations Based on the Y-Chromosome DNA Variation. Korean J Biol Sci 7: 139-144.

10. Jobling MA, Tyler-Smith C. 1995. Fathers and sons: the Y chromosome and human evolution. Trends Genet 11:449-456.

11. Kayser M, Krawczak M, Excoffier L, Dieltjes P, Corach D, Pascali V, Gehrig C, Bernini LF, Jespersen J, Bakker E, Roewer L, de Knijff P. 2001. An extensive analysis of Y-chromosomal microsatellite haplotypes in globally dispersed human populations. Am J Hum Genet 68:990 1018.

12. Kwak KD, Jin HJ, Shin DJ, Kim JM, Roewer L, Krawczak M, Tyler-Smith C, Kim W. 2005. Y-chromosomal STR haplotypes and their applications to forensic and population studies in East Asia. Int J Legal Med 119: 195-201.

13. Manni F., Leonardi P., Barakat A., Rouba H., Heyer E., Klintschar M., Mc Elreavey K., Quintana Murci L. 2002. Y-chromosome analysis in Egypt suggests a genetic regional continuity in Northeastern Africa. Hum Biol 74(5), 645-58.

14. Roewer L., Croucher PJP., Willuweit S., Lu TT., Kayser M., Lessig R., De Knijff P., Jobling MA., Tyler Smith C., Krawczak M. 2005. Signature of recent historical events in the European Y-chromosomal STR haplotype distribution. Hum Genet 116(4), 279-91.

15. Sambrook J, Fritsch EF, Maniatis T. 1989. Molecular cloning: A laboratory manual, 2nd edn. 
Cold Spring Harbor Laboratory Press, New York

16. Su B, Xiao J, Underhill P, Deka R, Zhang W, Akey J, Huang W, Shen D, Lu D, Chu J, Tan J, Shen P, Davis R, Cavalli-Sforza LL, Chakraborty R, Xiong M, Du R, Oefner P, Chen Z, Jin L. 1999. Y-chromosome evidence for a northward migration of modern humans into eastern Asia during the last Ice Age. Am J Hum Genet 65:1718-1724.

17. Underhill PA, Passarino G, Lin AA, Shen P, Lahr MM, Foley RA, Oeffner PJ, Cavalli-Sforza LL. 2001. The phylogeography of Y-chromosome binary haplotypes and the origins of modern human populations. Ann Hum Genet 65:43-62.

18. Zerjal T, Xue Y, Bortorelle G, Wells RS, Bao W, Zhu S, Qamar R, Ayub Q, Mohyuddin A, Fu S, Li P, Yuldasheva N, Ruzibakiev R, Xu J, Shu Q, Du R, Yang H, Hurles ME, Robinson E, Gerelsaikhan T, Dashnyam B, Mehdi SQ, Tyler-Smith C. 2003. The Genetic Legacy of the Mongols. Am J Hum Genet 72: 717-721. 